\title{
Instantaneous Datum Reconstruction Method of Multi-Beam Transducer in Short- Time GPS Signal Anomaly
}

\author{
Shengping Wang ${ }^{1,2,4}$, Jianmin Wang ${ }^{1,4}$, Ziyin $W_{u^{2, *}}$, Yongqi Wei ${ }^{3}$ and Liangyou Ouyang ${ }^{1,4}$ \\ ${ }^{1}$ Faculty of Geomatics, East China University of Technology, Nanchang 330013, China \\ ${ }^{2}$ Key Laboratory of Submarine Geosciences, Second Institute of Oceanography, Hangzhou 310012, China \\ ${ }^{3}$ School of Civil and Environmental Engineering, University of California, Los Angeles, California 951593, USA \\ ${ }^{4}$ Key Laboratory of Watershed Ecology and Geographical Environment Monitoring, East China University of Technology, \\ Nanchang 330013, China
}

Received 11 August 2017; Accepted 5 November 2017

\begin{abstract}
The short-time signal anomaly of Global Positioning System (GPS) often occurs in the process of multi-beam bathymetry, which leads to the poor accuracy of multi-beam acoustic emission of instantaneous position datum and affects the underwater topographic survey accuracy to a large extent. This study proposes a novel method to reconstruct multi-beam transducer instantaneous datum in the event of the short-time GPS signal anomaly to solve this problem. First, the synchronization of GPS and inertial measurement unit (IMU) was realized by establishing the correlation coefficient model. Second, the fast Fourier transformation filter was used to extract the long-period signal from the GPS elevation signal, which was repaired according to the Heave sequence, and the instantaneous elevation datum was reconstructed through attitude correction. Finally, the original plane information was obtained through Kalman filtering. The error accumulation of the original plane information was eliminated by using the fixed solution which had returned to normal, and then the instantaneous plane datum was reconstructed. The reliability of this method was proven through simulation experiments. Results demonstrate that the similarity between the GPS elevation sequence and Heave sequence can synchronize GPS and IMU. In addition, the instantaneous datum accuracy of the multi-beam transducer reconstructed through this method can reach the necessary cm-level to satisfy the requirements for the positioning accuracy in open water. The proposed method can establish a highly precise basis of data for the underwater seabed topographic monitor of signal anomaly areas in quayside and sea-crossing bridges.
\end{abstract}

Keywords: multi-beam transducer; instantaneous datum reconstruction; FFT low-pass filter; Kalman filtering

\section{Introduction}

With the rapid development of society and economy, the intense pressure of population, and the serious shortage of land resources, countries all over the world have successively shifted their development strategies to oceans, which have accounted for $71 \%$ of the world area. The multibeam measurement system is usually employed in the activities of marine resources development, marine science research, marine engineering construction, and national defense construction to obtain accurate seabed topography information. The instantaneous datum of multi-beam transducer is the basis for accurately obtaining the benthonic terrain information. However, the short-time Global Positioning System (GPS) signal anomaly ranging from a few seconds to a few minutes often occur during the measurement, which results in the instability or poor precision of the multi-beam transducer position datum (plane and elevation). Therefore, this study focuses on the precise instantaneous datum reconstruction method of multi-beam transducer in the short-term GPS signal anomaly based on the data provided by GPS, inertial measurement unit (IMU), and other auxiliary equipment of survey vessel.

*E-mail address: shpwang@ecit.cn

ISSN: 1791-2377 @ 2017 Eastern Macedonia and Thrace Institute of Technology. All rights reserved. doi:10.25103/jestr.106.10
Researchers have conducted numerous studies on GPS/IMU integrated navigation technology [1-5]. However, the low instantaneous datum accuracy of the multi-beam transducer during the GPS signals anomaly period remains. Therefore, establishing the relationship between GPS and IMU and realizing the data fusion method should be considered.

Three major problems regarding the instantaneous datum reconstruction of a multi-beam transducer must be solved. First, GPS and IMU must be synchronized for the time datum. However, the subsequent processing of the sensor data is not synchronized due to the influence of the GPS solution time, the radio transducer time, and the time difference of each sensor [6,7], thereby leading to the distortion of the underwater seabed topographic monitoring. In addition, the real-time kinematic (RTK) is a fixed solution when the GPS-RTK signal is good in open water; thus, RTK can be directly used to provide the elevation data for the transducer. However, obtaining direct position data is difficult because no solution exists for the short-time GPS signal anomaly. This accuracy is also difficult for the cmlevel, which is accuracy required for the underwater topographic survey. Finally, the plane position of the GPS in the signal anomaly period is obtained by interpolation processing during GPS signal anomaly, which will lead to the inaccuracy of the instantaneous plane datum of the multibeam transducer. 
Based on the preceding analysis, this study first establishes a time delay detection model based on the correlation coefficient method. Then, the reconstruction model of GPS elevation signal is reconstructed through the Heave sequence by studying the complementary characteristics of GPS and IMU. Finally, the characteristics of IMU accumulation error are analyzed through the Kalman filtering model. The results of the study will provide a reference for the multi-beam transducer instantaneous datum reconstruction during GPS signal anomaly.

\section{State of the art}

Introducing the study of the integrated navigation system of the land and the interior is necessary for the combination of GPS and IMU equipped on the survey vessel. Ibrahim et al. [8] studied indoor navigation by IMU and proved its reliability in the field. Park et al. [9] studied a low-cost sensor fusion method for vehicle location, which could adjust the noise covariance matrix $\mathrm{Q}$ and $\mathrm{R}$ to adapt to a variety of environments and complete the positioning task. However, the positioning accuracy is low in the case of the GPS signal anomaly. Liu et al. [10] presented a method which could effectively improve the inertial navigation system (INS)/GPS measurement accuracy by continuously updating the noise variance and collecting the estimates and measurements to deal with the noise variance of the system. The noise changes due to environmental disturbances could be treated by introducing attenuation factors and increasing the weight of current values. In addition, a few related defects exist in the transfer and utilization of land and indoor methods to the survey vessel. The ultra-wideband distance observation technique was introduced by Li et al. in [11]. for GPS/INS tightly-coupled navigation to improve the reliability and availability of GPS/INS integrated navigation in GPS-challenged environments. This method enhanced the stability of GPS/INS integrated navigation; however, the measurement error of this method was large when used on the multi-beam bathymetry due to the influence of seawater turbulence. Gao et al. [12] studied a novel approach by closely integrating the multi-global navigation satellite system (GNSS) single-frequency observation with IMU measurement. This method improved the accuracy, continuity, and reliability of precise point positioning (PPP); however, this method was invalid in the case of GPS signal anomaly. Khaghani et al. [13] used video display metafile to determine the availability of IMU data or the applicability of GNSS, whose results were more accurate than those of the traditional INS/GNSS navigation in the $5 \mathrm{~min}$ signal interruption period. Guo et al. [14] used a weighted combination filter for post-processing to improve the IMU/GPS accuracy, but the desired results can only be achieved at the premise of good GPS signals. Troiani et al. [15] studied the navigation method, which relies on the data provided by a single on-board camera and IMU in terms of insufficient GPS signals. However, the feature points cannot be located on the sea surface for image matching in the multi-beam measurement. Madeira et al. [16] used independent photogrammetric techniques to evaluate the accuracy of GNSS and IMU navigation; however, this method is only applicable to land. For time-delay detection combining GPS and IMU, Zhang et al. [17] proposed a multi-feature point matching delay detection technique based on the previous studies. The synchronization techniques of GPS and multi-beam depth sounder in the process of determining the instantaneous datum of multibeam transducer were studied; however, the time asynchronization of GPS and IMU was ignored. Liu [18] described the underwater topography measurement of GPS under no tidal mode in detail and studied the techniques of single characteristic point delay detection. Researchers have conducted numerous studies on the current problem of instantaneous datum determination of multi-beam transducers. Chang et al. [19] earlier proposed the concept that GPS can directly provide the elevation datum for multibeam transducers instead of tidal wave. Steven et al. [20] extracted tide-level data from the GPS-RTK signal through low-pass filters, and instead of shore station tidal elevation datum, GPS tidal data were directly used in this method as instantaneous transducer, thereby solving the difficulty of directly reflecting the vessel location tide on the coastal tide station data. The Ocean Mapping Group of UNB University in Canada has carried out many studies in this field since 2002. Among them, Clarke et al. [21] proposed a method for acquiring instantaneous elevation datum of transducer based on GPS-RTK and Heave fusion. The main measure of this method was to fuse the information of the stable lowfrequency information from the RTK fixed solution extracted by the fast Fourier transform (FFT) low-pass filter and the high-frequency information from the Heave sequence extracted by the FFT high-pass filter. Yang et al. [22] also analyzed the fusion accuracy of GPS and Heave and proved that the method could provide the instantaneous elevation of transducer with 6-7 cm accuracy, which was twice that of the traditional method. This method could achieve instantaneous height restoration of the transducer but would be invalid when RTK signal anomaly. Gandolfi et al. [23] studied GPS-PPP precision for short-time observation sessions and found that the precision of 1-h and a 1/2-h Receiver Independent Exchange Forma files was within $5 \mathrm{~cm}$ and $10 \mathrm{~cm}$, respectively.

The preceding studies mainly focus on excellent GPSRTK signal and the feature image matching. However, achieving high-precision navigation data on a single onboard GPS is difficult when GPS signals present anomaly. In addition, a few feature images matching on the sea surface exist, and navigation solely with IMU will lead to a considerable amount of error accumulation. Obtaining highprecision transducer instantaneous datum in the measurement process is impossible due to the preceding conditions. At present, literature on the instantaneous datum reconstruction of multi-beam transducer in short-term GPS signal anomaly is limited. According to the waveform similarity between GPS elevation signal and Heave signal, the correlation coefficient method is used to solve the time delay between GPS and IMU. An improved method based on Heave for short-term GPS signal anomaly is provided in this study to repair GPS elevation signal. This method uses the FFT low-pass filter to extract GPS low-frequency signal during the signal anomaly period and is complemented by the Heave sequence signal to complete the GPS elevation restoration during the abnormal period. Finally, we use the GPS fixed solution before short-time anomaly to provide the initial position for the IMU. The Kalman filtering model is established to obtain the optimal estimation of IMU position information and then remove the accumulation error by using the normal GPS fixed solution, thus redistributing multi-beam transducer instantaneous plane datum reconstruction.

The remainder of this study is organized as follows. The systematic model is established and the method for 
restructuring the instantaneous datum method is proposed in Section 3. Section 4 presents the discussion on the applicability and reliability of the method with a few examples and the analysis of test results. Section 5 summarizes the conclusions.

\section{Methodology}

\subsection{Time Synchronization}

GPS and IMU require time synchronization to achieve instantaneous reconstruction of the transducer [24]. As shown in Fig. 1, in terms of the original Heave sequence with GPS elevation sequence, "displacement phenomenon" often occurs due to the influence of time delay. This delay must be eliminated to obtain high-precision multi-beam transducer instantaneous datum.
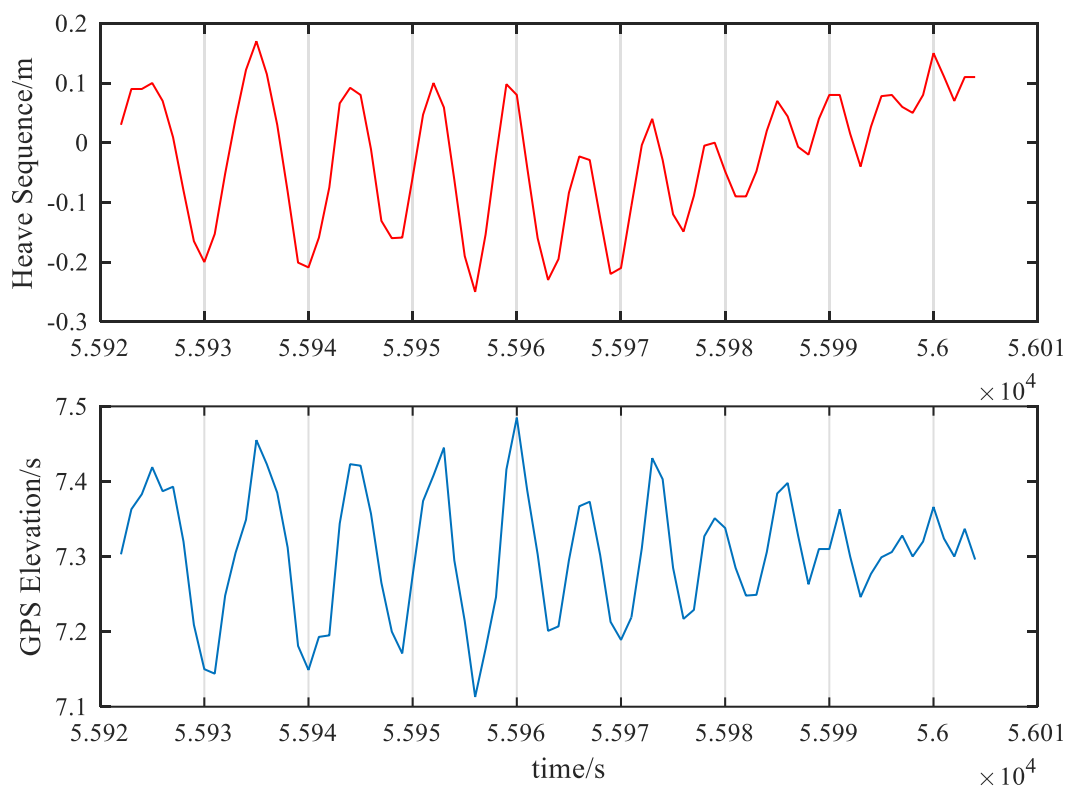

Fig. 1 Original GPS elevation and Heave comparison chart

The correlation coefficient method was used to determine time delay based on the correlation between the Heave sequence and the GPS elevation sequence. The GPS elevation sequence was taken as a benchmark, then the correlation coefficient was calculated whenever a small time was added to the Heave sequence, until the maximum correlation coefficient was obtained. Thus, the time delay between GPS and IMU equaled the total iteration time.

We selected a time series of evident elevation changes to work out the mathematical expectations. The Heave sequence mathematical expectation is $\bar{H}_{\text {Heave }}$, and the GPS elevation mathematical expectation is $\bar{H}_{G P S} \cdot H_{G P S}^{i}$ and $H_{H \text { eave }}^{i}$, which correspond to GPS elevation and Heave value at time $i$. Obtaining the correlation coefficient $k$ is based on Equation (1).

$k=\frac{\sum_{i=1}^{n}\left(H_{i}^{\text {Heave }}-\bar{H}_{\text {Heave }}\right)\left(H_{i}^{G P S}-\bar{H}_{G P S}\right)}{\sqrt{\sum_{i=1}^{n}\left(H_{i}^{\text {Heave }}-\bar{H}_{\text {Heave }}\right)^{2} \sum_{i=1}^{n}\left(H_{i}^{G P S}-\bar{H}_{G P S}\right)^{2}}}$

\subsection{Multi-beam transducer instantaneous elevation datum reconstruction}

\subsubsection{Improved method to repair GPS elevation sequence based on Heave sequence}

Signal anomaly often occurs in the process of GPS navigation as shown in Fig. 2(a), thereby leading to poor positioning accuracy. The GPS elevation signal must be corrected in the anomaly period to obtain precise elevation datum [25]. The Heave sequence can be used to correct the GPS elevation sequence because GPS and IMU simultaneously monitor the changes of the survey vessel in a vertical direction [26]. The calculation process is based on Equation (2).

$$
\begin{aligned}
& \Delta_{(i, i-1)}^{G P S}=H_{i}^{G P S}-H_{i-1}^{G P S}, \Delta_{(i, i+1)}^{G P S}=H_{i}^{G P S}-H_{i+1}^{G P S} \\
& \Delta_{(i, i-1)}^{\text {Heave }}=H_{i}^{\text {Heave }}-H_{i-1}^{\text {Heave }}, \Delta_{(i, i+1)}^{\text {Heave }}=H_{i}^{\text {Heave }}-H_{i+1}^{\text {Heave }} \\
& \delta_{1}=\Delta_{(i, i-1)}^{G P S}-\Delta_{(i, i-1)}^{\text {Heave }}, \delta_{2}=\Delta_{(i, i+1)}^{G P S}-\Delta_{(i, i+1)}^{\text {Heave }}
\end{aligned}
$$

According to the observation accuracy of IMU and GPS (the accuracy of Heave is $\pm 1 \mathrm{~cm}$, and the accuracy of GPS is $\pm 5 \mathrm{~cm}$ ), to set a threshold $\varepsilon$ (usually as $\pm 6 \mathrm{~cm}$ ). if $\delta_{1} \leq 2 \varepsilon$, then $H_{i}^{G P S}$ is normal; If $\delta_{1}>2 \varepsilon \& \delta_{2} \leq 2 \varepsilon$, then $H_{i}^{G P S}$ comes from Equation (3); if $\delta_{1}>2 \varepsilon \& \delta_{2}>2 \varepsilon$, then $H_{i}^{G P S}$ is derived from Equation (4).

$$
\begin{aligned}
& H_{i}^{G P S}=H_{i+1}^{G P S}+\Delta_{(i, i+1)}^{\text {Heave }} \\
& H_{i}^{G P S}=\left(\left(\mathrm{H}_{i-1}^{G P S}+\Delta_{(i, i-1)}^{\text {Heave }}\right)+\left(\mathrm{H}_{i+1}^{G P S}+\Delta_{(i, i+1)}^{\text {Heave }}\right)\right) / 2
\end{aligned}
$$

Equation (4) establish conditions that $H_{i-1}^{G P S}$ and $H_{i+1}^{G P S}$ are reliable value. In the actual measurement, the GPS signal anomaly often anomalies between a few seconds to a few minutes, making $H_{i-1}^{G P S}$ and $H_{i+1}^{G P S}$ unreliable.

Regarding this situation, this study recorded the anomalies of this period and deleted outliers. The long period signal of an entire GPS signal was extracted by FFT low-pass filter, and the Heave sequence of the time was added to complete the correction of GPS elevation signal.

FFT is a fast algorithm for implementing discrete Fourier transform (DFT) with few computations. The design idea of 
FFT low-pass filter is as follows: First, the original timedomain signals are transformed into frequency-domain signal by FFT. Second, the signal was converted to lowfrequency signal through the designed threshold that the high-frequency signal cannot pass through. Finally, the lowfrequency signals are converted to long period signals through inverse Fourier transform. The mathematical expression of the filter is shown in Equation (5).

$G(u, v)=H(u, v) F(u, v)$

In Equation (5), $G(u, v)$ is the filtered GPS elevation sequence, $F(u, v)$ is the input original time-domain signal,

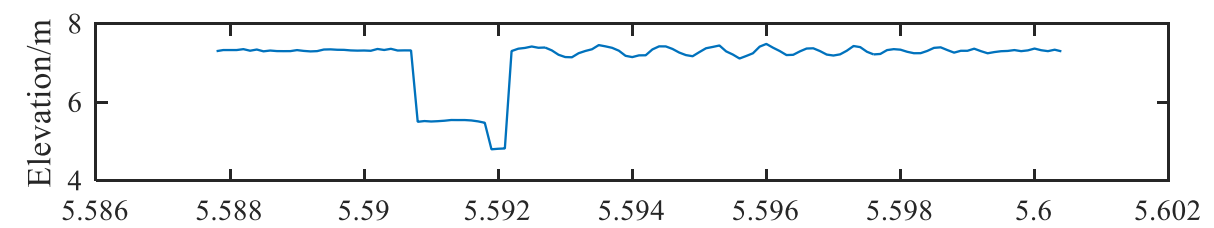

(a) Original GPS Elevation Sequence

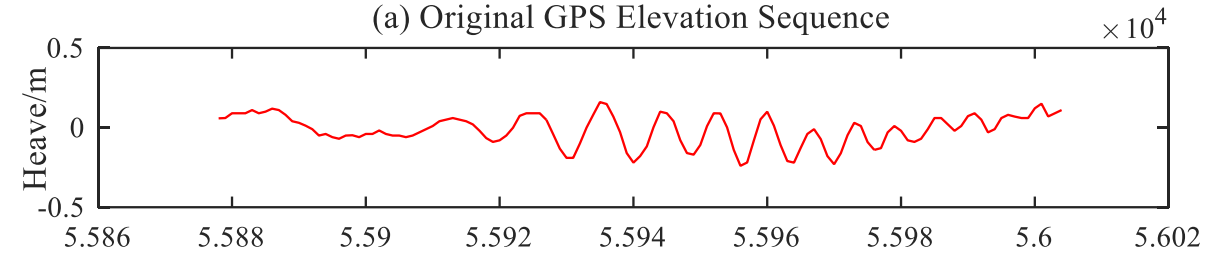

(b) Heave Sequence

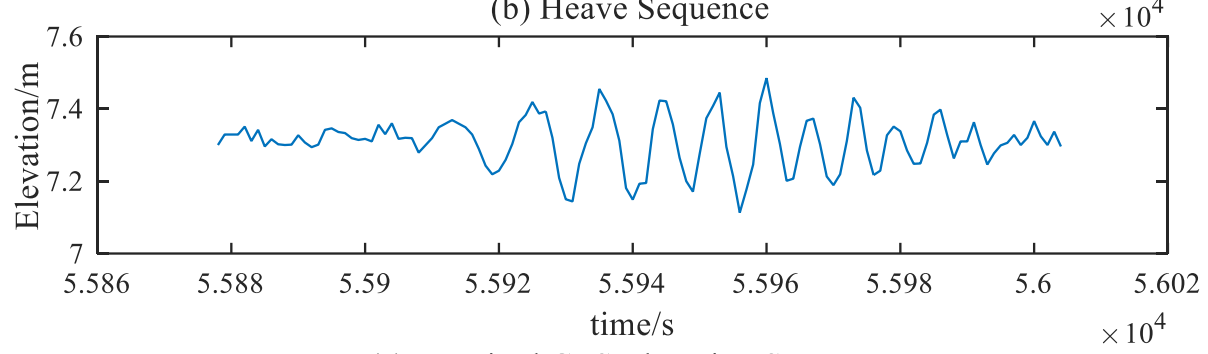

(c) Repaired GPS Elevation Sequence

Fig. 2 GPS elevation repair effort chart

\subsubsection{Attitude correction}

Owing to the influence of wind and waves, the survey vessel has a Roll $(r)$ around the X-axis, a Pitch $(p)$ around the y-axis, and a Heave $(h)$ in the vertical direction, which changes the antenna position of the GPS in the ideal vessel coordinate system. Therefore, attitude correction must be conducted to obtain the instantaneous elevation datum of the transducer [27].

Suppose the coordinate of GPS receiver in the ideal vessel coordinate system is $P(x, y, z)$, the attitude information is $(r, p, h)$, and the vertical distance is $h^{g}$ from GPS receiver antenna to transducer are known, attitude correction through Equation (7) can be allowed to obtain the coordinate $P\left(x_{0}, y_{0}, z_{0}\right)$ of the GPS receiver. Therefore, $H_{T}$ is the elevation of multi-beam transducer, which is calculated by Equation (8).

$$
\left[\begin{array}{l}
x \\
y \\
z
\end{array}\right]=R_{r} R_{p}\left[\begin{array}{l}
x_{0} \\
y_{0} \\
z_{0}
\end{array}\right]=\left[\begin{array}{ccc}
1 & 0 & 0 \\
0 & \cos r & \sin r \\
0 & -\sin r & \cos r
\end{array}\right]\left[\begin{array}{ccc}
\cos p & 0 & -\sin p \\
0 & 1 & 0 \\
\sin p & 0 & \cos p
\end{array}\right]\left[\begin{array}{l}
x_{0} \\
y_{0} \\
z_{0}
\end{array}\right]
$$

$H_{T}=Z-h^{g}$

\subsection{Multi-beam transducer instantaneous datum reconstruction}

and $H(u, v)$ is the transfer function of the filer. The FFT low-pass filter was used to suppress high-frequency signals, there are by enabling the extraction of long-period GPS elevation sequences.

The long period GPS elevation signal is $G(u, v)$, which was obtained through FFT low-pass filter at time $i$ of anomaly period. The Heave value is $H^{i}$ heave at time $i$. The point elevation is $H_{G P S}^{i}$, which is calculated by Equation (6).

$$
H_{G P S}^{i}=G^{i}(u, v)+H_{\text {Heave }}^{i}
$$

In GPS signal anomaly, the plane positioning data obtained by GPS in the survey vessel is unreliable, and using IMU navigation alone will result in poor accuracy due to the time longer of the IMU navigates, and the greater of the error accumulates. Since the short-time GPS signal anomaly often lasts time is short, then the error accumulates is small. Therefore, solving the problem of transducer plane position datum reconstruction under short-time GPS signal anomaly by studying the GPS and IMU interaction is possible.

The GPS signal will exhibit evident changes in its quality during the measurement process, such as lack of GPS signal, single-point solution, floating-point solution, and fixed solution. The preceding four states tend to suddenly switch to each other. Except for the fixed solution, the accuracy of the three remaining states cannot satisfy positioning requirements. The GPS positioning quality factors from the GPS data output format statement (\$GPGGA) are used to determine GPS signal status. The reconstruction work is completed through GPS/IMU data fusion in different environments [28].

When GPS signal is a fixed solution, the positioning accuracy satisfies the requirements of multi-beam underwater topographic measurement. Regarding short-time GPS signal anomaly, the IMU positioning error accumulation is small. The initial plane position information is obtained by building the Kalman filtering model with IMU. Then, the instantaneous plane datum reconstruction 
can be completed through the GPS fixed solution, which is correcting the error accumulation of initial plane position information.

Vector $\mathrm{x}$ and state vector $X=\left[x, v_{x}, a_{x}\right]$ has been observed to move in the $\mathrm{X}$ direction of IMU. The state and observation equations are established by Equations (9) and (10), respectively.

$$
\begin{aligned}
& {\left[\begin{array}{l}
x \\
v_{x} \\
a_{x}
\end{array}\right]_{k}=\left[\begin{array}{ccc}
1 & \Delta t & \Delta t^{2} / 2 \\
0 & 1 & \Delta t^{2} \\
0 & 0 & 1
\end{array}\right]\left[\begin{array}{c}
x \\
v_{x} \\
a_{x}
\end{array}\right]_{k-1}+\left[\begin{array}{c}
\Delta t^{3} / 6 \\
\Delta t^{2} / 2 \\
\Delta t
\end{array}\right] W_{K-1}} \\
& x_{k}=\left[\begin{array}{lll}
1 & 0 & 0
\end{array}\right]\left[\begin{array}{c}
x \\
v_{x} \\
a_{x}
\end{array}\right]_{k}+V_{k}
\end{aligned}
$$

Equations (9) and (10) can be discretized into Equations (11) and (12), respectively, because the data measured by the IMU are discrete.

$$
\begin{aligned}
& X_{k}=\Phi_{k / k-1} X_{k-1}+\Gamma_{k-1} W_{k-1} \\
& L_{k}=A_{k} X_{k}+V_{k}
\end{aligned}
$$

State equation and mean square error of one-step prediction equation of the Kalman filtering model can be established as shown in Equations (13) and (14), respectively [29].

$\hat{X}_{k / k-1}=\Phi_{k / k-1} \hat{X}_{k-1}$

$P_{k / k-1}=\Phi_{k / k-1} P_{k-1} \Phi_{k / k-1}^{T}+\Gamma_{k-1} Q_{k-1} \Gamma_{k-1}^{T}$

Gain is computed through Equation (15).

$$
K_{k}=P_{k / k-1} A_{k}^{T}\left(A_{k} P_{k / k-1} A_{k}^{T}+R_{k}\right)^{-1}
$$

State estimation is computed through Equation (16), and mean square error is computed through Equation (17).

$$
\begin{aligned}
& \hat{X}_{k-1}=\hat{X}_{k / k-1}+K\left(L_{k}-A_{k} X_{k / k-1}\right) \\
& P_{k}=\left(I-K_{k} A_{k}\right) P_{k / k-1}
\end{aligned}
$$

Where $X_{k}$ is the state vectors at time $k$, and $X_{k-1}$ is the state vectors at time $k-1 ; \Phi_{k / k-1}$ is the state transition matrix, $W_{k-1}$ is the system noise coefficient matrix, and $\Gamma_{k-1}$ is the noise coefficient matrix at time $k-1 . L_{k}$ is the observation vector, $A_{k}$ is the observation matrix, and $V_{k}$ is observation noise at time $k . Q_{k}$ is variance matrix of the observation noise, and $R_{k}$ is variance matrix of system noise. $K$ is the gain matrix at time $k$, and $P_{k}$ is the variance matrix of the state estimate at time $k$.

According to the one-step Kalman filtering model, the entered initial value and initial covariance matrix and the imported state vectors are used to complete the optimal estimation of observations through continuous iteration and then error distribution is carried out. The initial design of the error distribution method is based on the notion that distance is proportional to time to distribute error. Suppose that coordinate $\mathrm{X}$ is $X_{0}$ before entering the blind area, and the first fixed solution is $X_{G P S}$ after the blind area. The coordinate is $X_{I M U}$ by the Kalman filtering model calculation, and each distance is $\Delta X_{i}$ at time $i$. We compute $\mathrm{X}$ coordinate at each moment as $x_{i}$ at time $i$.

$x_{i}=X_{0}+\sum_{i}^{i}\left(\Delta X_{i}-\frac{\Delta X_{i}\left(X_{I M U}-X_{G P S}\right)}{\left(X_{I M U}-X_{0}\right)}\right)$

The preceding methods are used to reconstruct $\mathrm{X}$ - and $\mathrm{Y}$ axis coordinates. The tracks of transition and reconstruction are shown in Fig. 3.
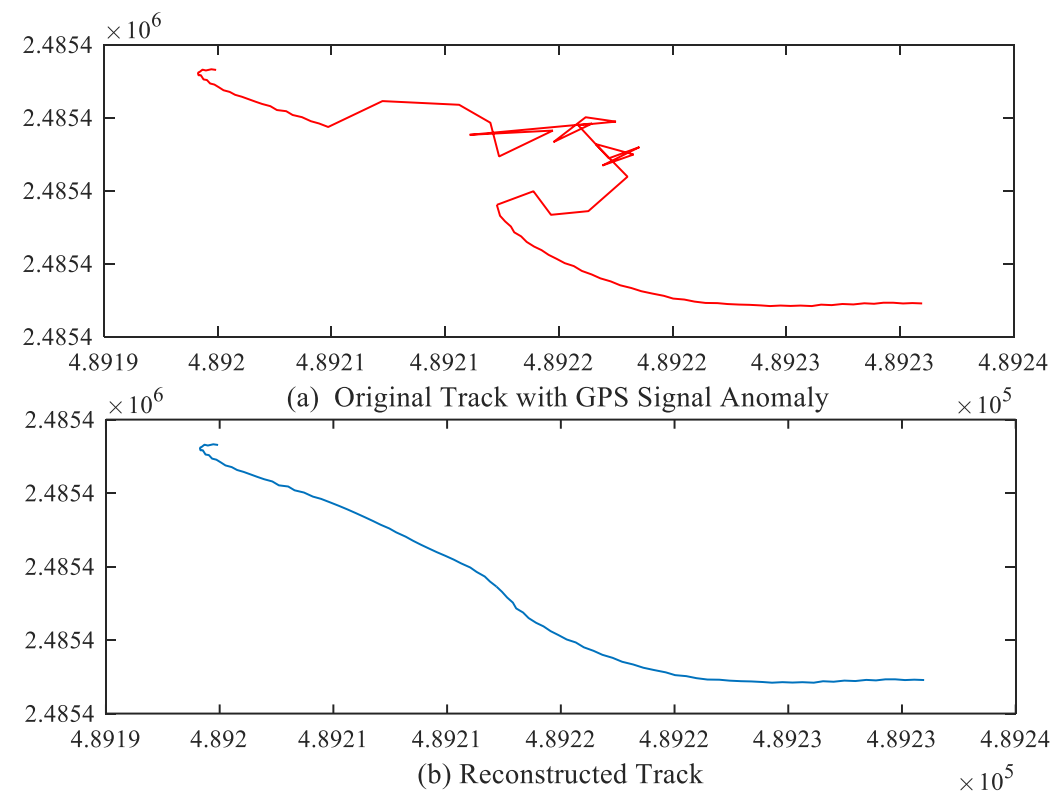

Fig. 3 Track comparison chart 


\section{Result Analysis and Discussion}

The simulation experiment, which is conducted to verify the accuracy and reliability of multi-beam transducer datum reconstruction under short-time GPS signal anomaly, is based on the South Lingrui S86 GPS receiver of the RTK model. Receiver sampling frequency is set to $1 \mathrm{~s}$, and GPS antenna is installed in an open position of the vessel. IMU is installed at the vessel's center of gravity, with a sampling frequency of $50 \mathrm{HZ}$ and an accelerometer error of $0.002 \mathrm{~g}$. The experiment is performed with a piece of Simulation short-time GPS signal anomaly data that are measured in open water.

\subsection{Time synchronization correlation analysis}

Based on the methods of section 3.1, we set initial translation as $0.1 \mathrm{~s}$ to obtain the initial maximum correlation coefficient of 0.8626 at the fifth iteration. We set precise translation as $0.01 \mathrm{~s}$ to continue iteration from $0.4 \mathrm{~s}$ to $0.5 \mathrm{~s}$ and obtain the maximum correlation coefficient of 0.8639 at $0.45 \mathrm{~s}$. Therefore, the time delay between the Heave sequence and the GPS elevation sequence is $0.45 \mathrm{~s}$.

The comparison between the Heave sequence and the GPS elevation sequence presented in Fig. 4 shows that these sequences are basically synchronized by the correlation coefficient method discussed in Section 3.1. Thus, subsequent work on the instantaneous datum reconstruction of multi-beam transducers can be performed.
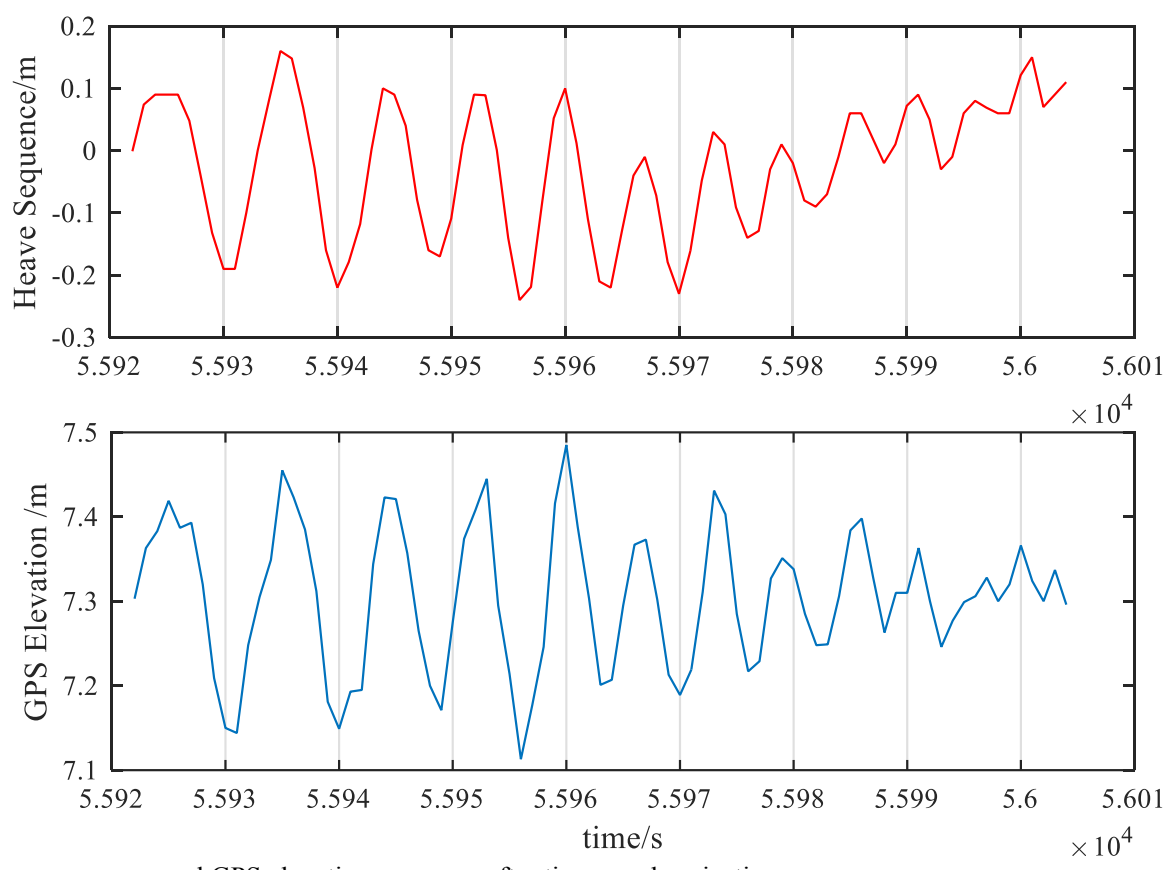

Fig. 4 Comparison of Heave sequence and GPS elevation sequence after time synchronization

Table 1. Translation volume and corresponding correlation coefficient statistics

\begin{tabular}{c|c|c|c|c|c|c|c}
\hline $\begin{array}{c}\text { Initial } \\
\text { translation/s }\end{array}$ & $\begin{array}{c}\text { Correlation } \\
\text { coefficient }\end{array}$ & $\begin{array}{c}\text { Initial } \\
\text { translation/s }\end{array}$ & $\begin{array}{c}\text { Correlation } \\
\text { coefficient }\end{array}$ & $\begin{array}{c}\text { Precision } \\
\text { translation/s }\end{array}$ & $\begin{array}{c}\text { Correlation } \\
\text { coefficient }\end{array}$ & $\begin{array}{c}\text { Precision } \\
\text { translation/s }\end{array}$ & $\begin{array}{c}\text { Correlation } \\
\text { coefficient }\end{array}$ \\
\hline 0 & 0.8094 & 0.5 & 0.8626 & 0.41 & 0.8625 & 0.46 & 0.8634 \\
0.1 & 0.8320 & 0.6 & 0.8563 & 0.42 & 0.8631 & 0.47 & 0.8630 \\
0.2 & 0.8454 & 0.7 & 0.8425 & 0.43 & 0.8634 & 0.48 & 0.8626 \\
0.3 & 0.8584 & 0.8 & 0.8311 & 0.44 & 0.8635 & 0.49 & 0.8614 \\
0.4 & 0.8605 & 0.9 & 0.8079 & 0.45 & 0.8637 & & \\
\hline
\end{tabular}

4.2 Analysis on multi-beam transducer instantaneous elevation datum reconstruction simulation experiment The experiment simulated short-time GPS signal anomaly for $20 \mathrm{~s}$, with a threshold set to $3 \mathrm{~cm}$. The method used to repair the simulated GPS signal anomaly is shown in Section 3.2.1, and the experimental results are shown in Fig. 5.

The original GPS elevation without the anomaly shown in Fig. 5(a), while the simulated short-time GPS signal anomaly reflected by elevation changes from $4.7 \mathrm{~m}$ to $7.8 \mathrm{~m}$ shown in Fig. 5(b). The considerable change of approximately $3.1 \mathrm{~m}$ is not in line with the actual change in short times. However, the repaired elevation signal of the anomaly period of the investigated method is in line with the actual signal. The maximum change of the curve is $0.4 \mathrm{~m}$, thereby ranging from $7.1 \mathrm{~m}$ to $7.5 \mathrm{~m}$, and the maximum deviation is $4.5 \mathrm{~cm}$, as shown in the Fig. 5(c). These results are consistent with the original signal.

Attitude correction is also used to eliminate the influence of wind and waves, obtained highly precise the transducer instantaneous elevation datum is shown in Fig. 6. 
Shengping Wang, Jianmin Wang, Ziyin Wu, Yongqi Wei and Liangyou Ouyang/

Journal of Engineering Science and Technology Review 10 (6) (2017) 70-78

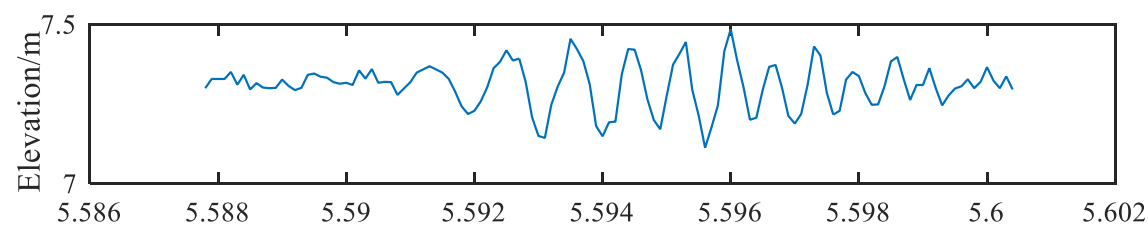

(a) Original GPS Elevation Sequence

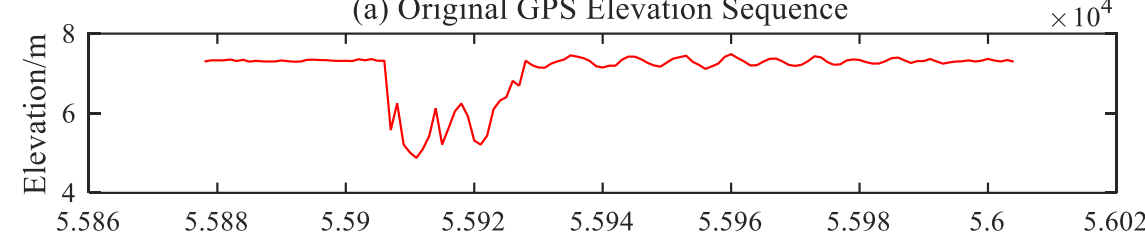

(b) Simulated GPS Elevation Sequence with Signal Anomaly $\times 10^{4}$

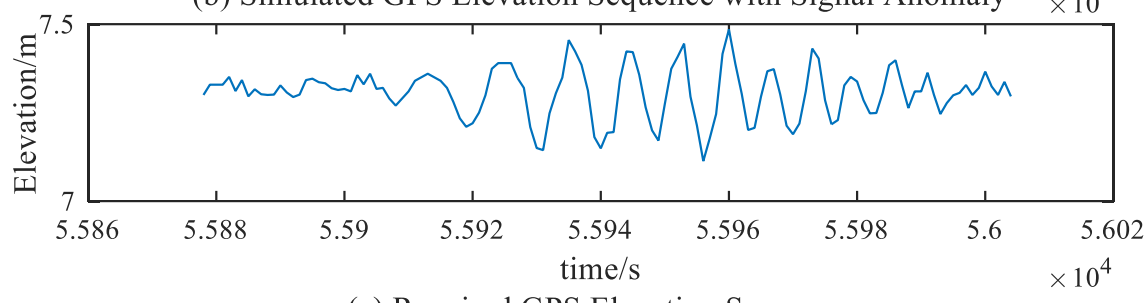

(c) Repaired GPS Elevation Sequence

Fig. 5 GPS elevation correction before and after the comparison map in simulation experiment
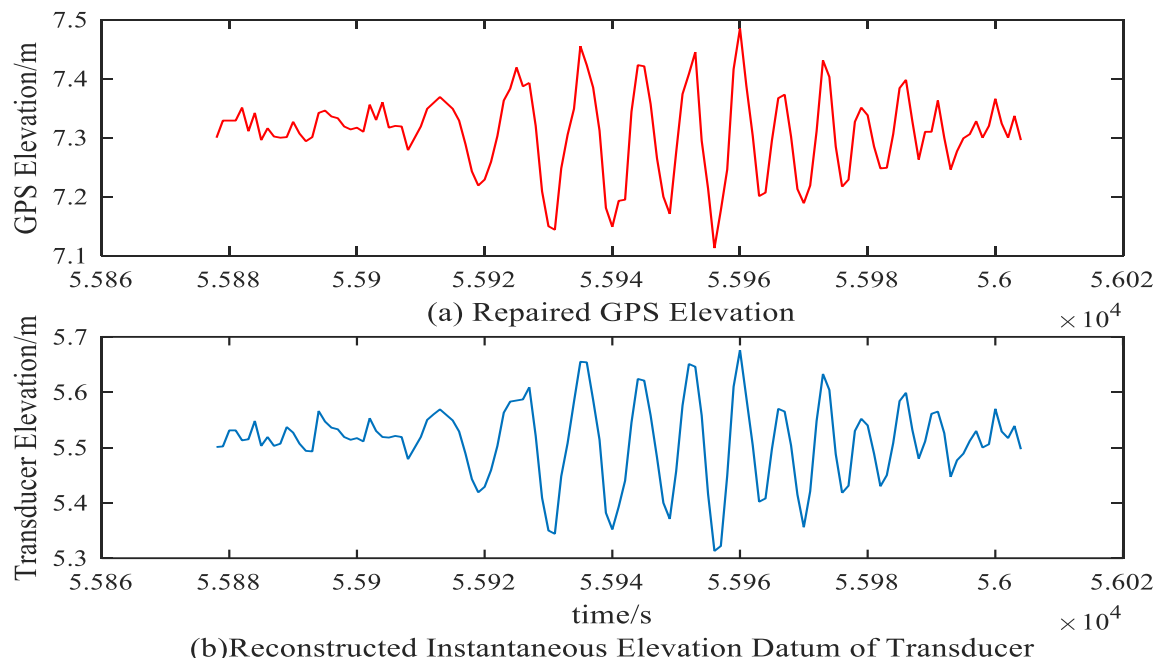

Fig. 6 reconstruct of the instantaneous elevation of the transducer effort chart

4.3 Analysis on multi-beam transducer instantaneous plane datum reconstruction simulation experiment

A piece of data with a straight line was selected in this study to simulate the plane information of the GPS signal anomaly. The information of the plane position in this period was reconstructed by the method presented in Section 3.3. The obtained results are shown in Figs. 7 and 8.

The original $\mathrm{X}$ and $\mathrm{Y}$ coordinates are straight lines because the original track line is also a straight line. By simulating the anomaly, the $\mathrm{X}$ and $\mathrm{Y}$ coordinates are became curved, which are inconsistent with the actual track. After reconstruction, the $\mathrm{X}$ and $\mathrm{Y}$ coordinates appeared to be similar to the original coordinates.

The multi-beam transducer instantaneous plane datum under short-term GPS signal anomaly simulation was reconstructed with the proposed methods in this study. Taking the GPS data under normal conditions as reference and then conducting a statistical analysis, the $\mathrm{X}$-coordinate maximum error was $3.4 \mathrm{~cm}$, and the $\mathrm{Y}$-coordinate maximum error was $2.9 \mathrm{~cm}$, as shown in Table 2 . All deviations are cm-level.

Table 2. Accuracy statistics for instantaneous datum reconstruction of multi-beam transducers

\begin{tabular}{c|c|c|c|c}
\hline Experiment & $\begin{array}{c}\text { Maximum deviation } \\
(\mathbf{c m})\end{array}$ & $\begin{array}{c}\text { Minimum deviation } \\
(\mathbf{c m})\end{array}$ & $\begin{array}{c}\text { Deviation average } \\
(\mathbf{c m})\end{array}$ & Standard deviation (cm) \\
\hline $\boldsymbol{x}$ coordinate & 3.4 & 0.2 & 0.2 & 1.6 \\
$\boldsymbol{y}$ coordinate & 2.9 & 0.2 & 0.2 & 1.5 \\
\hline
\end{tabular}




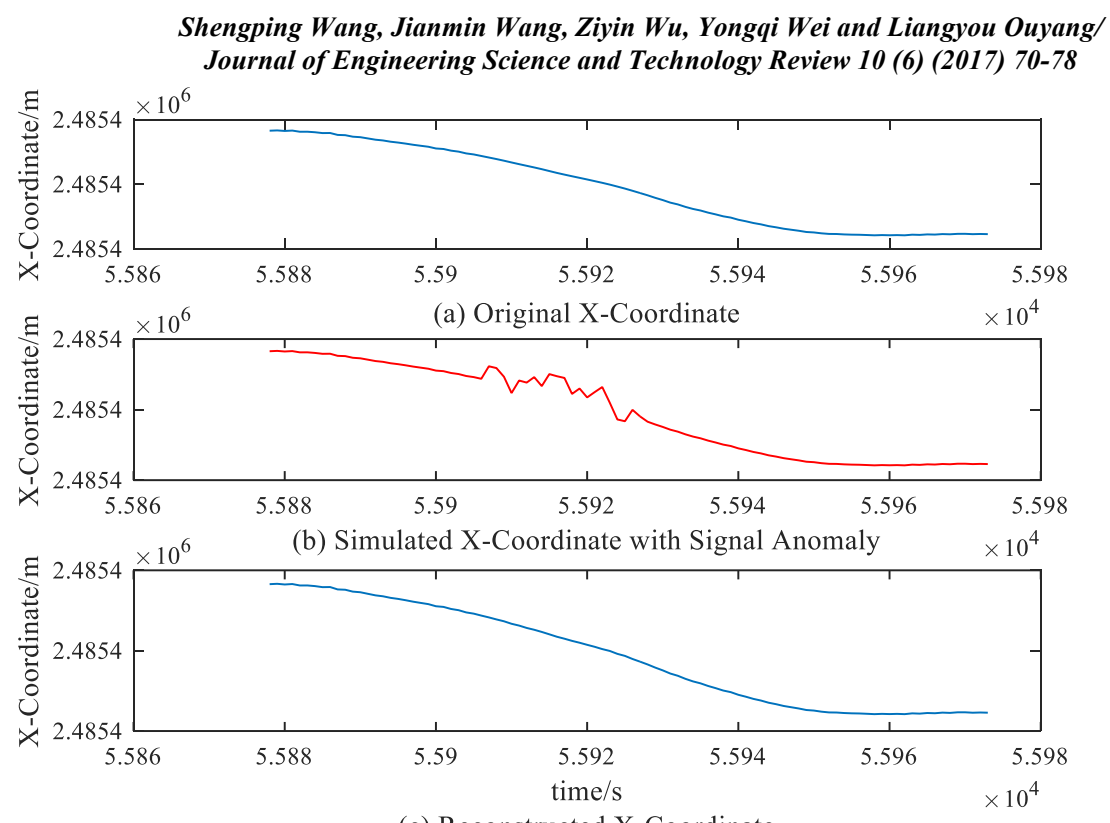

(c) Reconstructed X-Coordinate

Fig. 7 x-coordinate reconstruction before and after the comparison map in simulation experiment
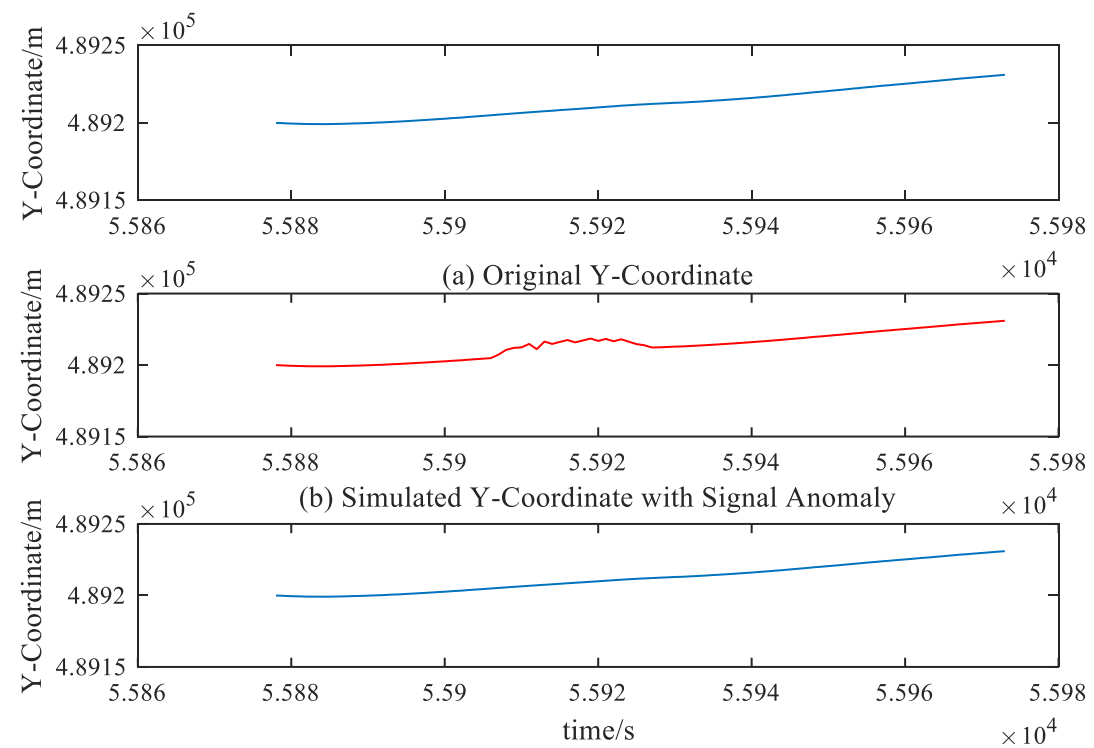

(c) Reconstructed Y-Coordinate

Fig. 8 y-coordinate reconstruction before and after the comparison map in simulation experiment

\section{Conclusions}

A novel method based on GPS/IMU was developed to reconstruct the instantaneous datum of multi-beam transducer to solve the poor accuracy of transducer instantaneous datum under short-time GPS signal anomaly. The combination of numerical simulation and experimental research method was used to analyze instantaneous datum accuracy of transducer reconstruction. The following results were obtained:

(1) The correlation coefficient method was used to obtain high accuracy time delay based on obvious similarities between GPS elevation and Heave sequence waveform.

(2) Repairing the GPS elevation based on the conventional methods in short-time GPS signal anomaly is not feasible, but the FFT low-pass filter can be used to effectively repair the GPS signal anomaly.

(3) In the short GPS signal anomaly period, IMU must be used for navigation. Setting time to eliminate the error accumulation of IMU information can improve the instantaneous plan datum accuracy well.

A combination of theory and experimental method is used to study the instantaneous reconstruction method of the multi-beam transducer during the short-time GPS signal anomaly period. The high accuracy satisfies the requirements of no tidal mode underwater topographic survey in the open water patterns with its cm-level accuracy. However, the proposed method is only applicable in the case of short-time GPS signal anomaly. Therefore, future research can focus on correcting multi-beam transducer instantaneous datum in the long-period GPS signal anomaly.

\section{Acknowledgements}

This research work was supported by t the Doctor Start-up Fund of the East China University of Technology (DHBK2012204), the National Natural Science Foundation of China (41206078, 41404026), Natural Science Foundation of Jiangxi Province (20161BAB206163), the Director Fund of the Second Institute of Oceanography (JG1508) and Graduate Innovation Fund of the East China University of Technology (DHYC-2017006). 
This is an Open Access article distributed under the terms of the Creative Commons Attribution License

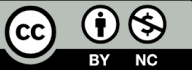

\section{References}

1. Yang, Y. X., "Study of IMU/GPS Composed Navigation System for Shipboard UAV". Ship Electronic Engineering, 36(2), 2016, pp.31-33.

2. Liu, Q. Y., Hao, L. L., Huang, S. J., "A New Study of Neural Network Aided GPS/MEMS-INS Integrated Navigation". Journal of Geomatics Science and Technology, 31(4), 2014, pp.336-341.

3. Saadeddin, K., Abdel-Hafez, M. F., Jaradat, M. A., Jarrah, M. A., "Performance enhancement of low-cost, high-accuracy, state estimation for vehicle collision prevention system using ANFIS". Mechanical systems and Signals Processing, 41(1-2), 2013, pp.239-253.

4. Alam, N., Kealy, A., Dempster, A. G., "Cooperative Inertial Navigation for GNSS-Challenged Vehicular Environments". IEEE Transactions on Intelligent Trandportation Systems, 14(3), 2013, pp.1370-1379.

5. Roberts, A., Tayebi, A., "A new position regulation strategy for VTOL UAVs using IMU and GPS measurements". Automatica, 49(2), 2013, pp. 434-440.

6. Ganeriwal, S., Kumar, R., Srivastava, M. B., "Timing-sync protocol for sensor networks". In: Proceedings of theFirst ACM Conference on Embedded Networked Sensor Systems, Los Angeles, USA: ACM Press, 2003, pp.138-149.

7. Elson, J., Girod, L., Estrin, D., "Fine-Grained Network Time Synchronization using Datum Broadcasts". In: Proceedingsof the 5th Symposium on Operating Systems Design and Implementation, New York, USA: ACM Press, 2002, pp.147163.

8. Ibrahim, M., Moselhi, O., "Inertial measurement unit based indoor localization for construction applications". Automation Inconstruction, 71(1), 2016, pp.13-20.

9. Park, G. H., Wang, Y., Choi, S. B., "Vehicle Positioning Based on Velocity and Heading Angle Observer Using Low-Cost Sensor Fusion”. Journal of Dynamic Systems Measurement and Control, 139(12), 2017, pp.1-13

10. Li, Y. H., Fan, X. Q., Lv, C., Wu, J., Li, L., Ding, D. W., “An innovative information fusion method with adaptive Kalman filter for integrated INS/GPS navigation of autonomous vehicles". Mechanical Systems and Signal Processing, 100(1), 2017, pp.605-616.

11. Li, Z. K., Chang, G. B., Gao, J. X., Wang, J., Hernandez, A.,"GPS/UWB/MEMS-IMU tightly coupled navigation with improved robust Kalman filter". Advances in Space Research, 58(11), 2016, pp.2424-2434

12. Gao, Z. Z., Ge, M. R.., Shen, W. B., Zhang, H. P., Niu, X. J., "Ionospheric and receiver DCB-constrained multi-GNSS singlefrequency PPP integrated with MEMS inertial measurements". Journal of Geodesy, 91(11), 2017, pp.1351-1366.

13. Khaghani, M., Skaloud, J., "Autonomous Vehicle Dynamic Model-Based Navigation for Small UAVs". Navigation-Journal of the Institute of Navigation, 63(3), 2016, pp.345-358.

14. Guo, H., Guo, J., Yu, M., Hong, H. B., Xiong, J., "A weighted combination filter with nonholonomic constrains for integrated navigation systems". Advances in Space Research, 55(2), 2015, pp.1470-1476
15. Troiani, C., Martinelli, A., Laugier, C., Scaramuzza, D., "Low computational-complexity algorithms for vision-aided inertial navigation of micro aerial vehicles". Robotics and Autonomous Systems, 69(1), 2015, pp.80-97.

16. Madeira, S., Yan, W. L., Bastos, L., Goncalves, J. A., "Accuracy Assessment of the Integration of GNSS and a MEMS IMU in a Terrestrial Platform". Sensors, 14(11), 2014, pp.20866-20881.

17. Zhao, J. H., Zhang, H. M., Clarke. J. E. H., "Determination of Precise Instantaneous Tidal Level at Vessel". Geomatics and Information Science of Wuhan University, 31(12), 2006, pp.10671070 .

18. Liu, Y. C., "Space Structure and Data Processing in Marine Sounding", Beijing: Survey and Mapping Press, China, 2003, pp.120-125.

19. Chang, C.C., Lee, H. W., "Preliminary test of Tide-Independent Bathymetric Measurement Based on GPS". Geomatics Research Australasia, 76(1), 2002, pp.23-36.

20. Zhao, J. H., Brucker, S., Clarke, J. E. H., Duffy, G., “On the Fly GPS Tide Measurement along the Saint John River". International Hydrographic Review, 5(3)2004, pp.48-58.

21. Brucker, S., Clarke, J. H., Beaudoin, J., Lessels, C., Czotter, K. Loschiavo, R., Iwanowska, K., Hill, P., "Monitoring flood-related change in bathymetry and sediment distribution over theSquamish Delta". In: Hydrographic Conference Proceedings, British Columbia, Canada: IEEE, 2011, pp.1-16.

22. Yang, F. L., Zhao, J. H., Zhang, H. M., Han, X. D., "Blend of RTK Height Signal and Heave Signal and Accuracy Analysis". Geomatics and Information Science of Wuhan University, 31(3), 2007, pp.226-228

23. Gandolfi, S., Tavasci,L., Poluzz, L., "Study on GPS-PPP precision for short observation sessions". GPS Solutions, 21(3), 2017, pp.887-896.

24. Gui, X., Qin, H. B., Wang, S. P., Chen, X. Y., "A Time-delay Detection Method of Underwater Topographic Measurement Based on Correlation Coefficient and Iteration Method". Bulletin of Surveying and Mapping, 5(1), 2015, pp.57-59.

25. Zhao, J. H., Zhang, H. M., Clarke, J. E. H., Wang, S. P., "Determination of Precise Instantaneous Height at Multi-beam Transducer". Geomatics and Information Science of Wuhan University, 31(11), 2006, pp.983-986.

26. Zhao, J. H., Liu, J. N., "Multi - beam sounding and image data processing". Wu Han: Wuhan University Press, China, 2008 pp.178-179.

27. Zhao, J. H., Liu, J. N., Zhang, H. M., "The Analysis of Vessel Attitude and the Effect for Multibeam Echo Sounding". Geomatics and Information Science of Wuhan University, 25( 4), 2000, pp. 353-357.

28. Hao, H. J., Qin, C. Y., "GPS/IMU Data Fusion in Different Environments". Journal of Qingdao University(Engineering \& Technology Edition), 24(2), 2009, pp.67-71.

29. Wu, J. H., Zhang, H. C., "Application of adaptive Kalman filter based on contextual information to integrated GPS/IMU navigation". Application Research of Computers, 26(10), 2009, pp.3840-3842. 